\title{
PUZZLING THE MIND: EVALUATING THE DIFFICULTY OF GENERATED PUZZLE GAME LEVELS FOR A PUZZLE GAME INTERVENTION - PRELIMINARY RESULTS
}

\author{
Alvin Chesham ${ }^{1}$, Stephan Moreno Gerber ${ }^{1}$, Narayan Schütz ${ }^{1}$, Patric Wyss ${ }^{1}$, Prabitha
} Urwyler $^{1,2}$, Klemens Gutbrod ${ }^{3}$, Rene Müri ${ }^{4}$, Tobias Nef ${ }^{5}$

${ }^{1}$ Gerontechnology and Rehabilitation Group, University of Bern, Bern, Switzerland;

${ }^{2}$ University Hospital of Old Age Psychiatry, Bern, Switzerland

${ }^{3}$ Bern University Hospital Inselspital, Bern, Switzerland;

${ }^{4}$ Department of Cognitive and Restorative Neurology, Bern University Hospital Inselspital, Bern, Switzerland;

${ }^{5}$ ARTORG Center for Biomedical Engineering Research, University of Bern, Bern,

Switzerland.

Contact e-mail: alvin.chesham@ artorg.unibe.ch

Background: Engagement in cognitively stimulating leisure activities plays an important role in mediating cognitive decline. Recent studies suggest that computerized puzzle games are an activity liked by older adults that offers cognitive benefits. For use as a cognitive intervention, however, it is crucial that the puzzle game difficulty is constantly adapted to match the player's level of skill. To this end, we generated large sets of difficulty levels for two tablet-based puzzle games.

Methods: We conducted a play-test study in 16 healthy younger adults (18 to 40 years) that played and rated the difficulty of sets of levels for two board-based puzzle games: A Match-3 puzzle game (40 levels) that is played by swapping two objects to make a row of three identical objects, and a Numberlink puzzle game (55 levels) where paths to connect pairs of objects on the board must be found. For both puzzle games, sets of difficulty levels were generated using Python scripts by varying puzzle variables, that is the size of the puzzle board (all combinations of width and height from 4 to 8 ) and the number of game objects (from 4 to 8). For each level played, a subjective difficulty rating (from 1 to 10) and puzzle solving time were recorded. Furthermore, participants completed neurocognitive tests that measure cognitive domains engaged by the puzzle games and general cognitive ability.

Results: Mixed model analyses showed significant effects of both puzzle board size and number of game objects on puzzle solving time for both puzzle games. For puzzle difficulty ratings, however, there was only a significant effect of puzzle board size.

Conclusions: Our results indicate that puzzle difficulty can be manipulated by varying puzzle variables. In a next step, we will test more participants, including healthy older adults (above 65 years), to further examine whether age and cognitive ability are associated with performance on the two puzzle games. Lastly, this study will allow us to develop an algorithm for dynamic puzzle difficulty adjustment in a clinical trial using the two puzzle games as a cognitive intervention. 